\title{
Determining the cosmic ray ionization rate in dynamically evolving clouds
}

\author{
C. J. Lintott and J. M. C. Rawlings
}

Department of Physics and Astronomy, University College London, Gower Street, London, WC1E 6BT, UK

e-mail: cjl@star.ucl.ac.uk

Received 15 July 2005 / Accepted 1 November 2005

\section{ABSTRACT}

The ionization fraction is an important factor in determining the chemical and physical evolution of star forming regions. In the dense, dark starless cores of such objects, the ionization rate is dominated by cosmic rays; it is therefore possible to use simple analytic estimators, based on the relative abundances of different molecular tracers, to determine the cosmic ray ionization rate. This paper uses a simple model to investigate the accuracy of two well-known estimators in dynamically evolving molecular clouds. It is found that, although the analytical formulae based on the abundances of $\mathrm{H}_{3}^{+}, \mathrm{H}_{2}, \mathrm{CO}, \mathrm{O}, \mathrm{H}_{2} \mathrm{O}$ and $\mathrm{HCO}^{+}$give a reasonably accurate measure of the cosmic ray ionization rate in static, quiescent clouds, significant discrepancies occur in rapidly evolving (collapsing) clouds. As recent evidence suggests that molecular clouds may consist of complex, dynamically evolving sub-structure, we conclude that simple abundance ratios do not provide reliable estimates of the cosmic ray ionization rate in dynamically active regions.

Key words. astrochemistry - stars: formation

\section{Introduction}

In this paper we examine the accuracy of the methods used to constrain the rate of cosmic-ray induced ionization in molecular clouds. The level of ionization within a dark cloud may determine whether or not the cloud is stable against gravitational collapse leading to the formation of proto-stellar objects. This is particularly true if magnetic fields permeate the cloud - in which case the process is controlled by the coupling between the ions (tied to the magnetic field lines) and the neutral species. This applies both to quasi-static contraction (via ambipolar diffusion) and turbulent dissipation. Ruffle et al. (1998), investigated these processes and showed that the stability of low extinction $\left(A_{\mathrm{v}}<3\right)$ clumps in the Rosette Molecular Cloud could be explained in terms of photochemically driven high ionization levels, which inhibit the damping of (MHD) turbulent support.

Cosmic rays also play a very important rôle in the physics of the interstellar medium - for example, they are an important source of heating in dark molecular clouds (e.g. Goldsmith $\&$ Langer 1978). These dark clouds are highly opaque to optical and ultraviolet (ionizing) interstellar radiation, yet they are known to contain a wide variety of atomic and molecular ions. Although dust grain surface chemistry is extremely important in dark and diffuse clouds (most significantly in the synthesis of $\mathrm{H}_{2}$ ) the chemistry within dark clouds is driven by gasphase reactions and the most significant of these (particularly for oxygen and nitrogen-bearing species) are ion-neutral reactions. The source of this ionization is believed to be cosmic rays. In the case of diffuse media the initiating reaction is

$\mathrm{H}+\mathrm{crp} \longrightarrow \mathrm{H}^{+}+\mathrm{e}^{-}$

(where crp stands for "cosmic ray particle")

whilst in dense molecular clouds approximately $97 \%$ of cosmic ray- $\mathrm{H}_{2}$ impacts lead to the formation of $\mathrm{H}_{2}^{+}$(Hartquist $\&$ Williams 1995) via the reaction:

$\mathrm{H}_{2}+\mathrm{crp} \longrightarrow \mathrm{H}_{2}^{+}+\mathrm{e}^{-}$

which is followed by the rapid reaction:

$\mathrm{H}_{2}^{+}+\mathrm{H}_{2} \longrightarrow \mathrm{H}_{3}^{+}+\mathrm{H}$.

Subsequently, the $\mathrm{H}_{3}^{+}$drives ion-molecule chemistries (see below). The rate of ionization and electron abundance within these clouds therefore has enormous implications for the efficiency of the molecular chemistry and the overall physical stability of many dark clouds.

As a result of these reactions, nearly every cosmic-ray ionization creates an $\mathrm{H}_{3}^{+}$ion and this reacts with an abundant metal, such as oxygen or nitrogen (see below). The rate coefficient for reaction (1), $\zeta$, is therefore a key parameter in models of dark cloud chemistry; the relevant timescale for a cosmic-ray ionization induced chemistry to reach equilibrium is of the order of the fractional abundance of the metals (approximately $10^{-4}$ ) divided by $\zeta$. A value of $\zeta=1.3 \times 10^{-17} \mathrm{~s}^{-1}$ is often quoted in the literature, although in most cases only 
an order of magnitude accuracy is appropriate. In some sources, much higher values of $\zeta$ have been inferred (e.g. at least $\zeta=8 \times$ $10^{-16} \mathrm{~s}^{-1}$ in $o$ Per, van Dishoeck \& Black 1986). Generally, studies typically indicate that $\zeta=10^{-16}-10^{-17} \mathrm{~s}^{-1}$ per hydrogen atom.

However, if we assume a "standard" value of $\zeta \sim 10^{-17} \mathrm{~s}^{-1}$, the chemical timescale is approximately $10^{6}$ years. Usually, this assumption of equilibrium is adopted when using molecular abundances to infer the value of $\zeta$, but here we note that the free-fall collapse timescale of a gas cloud is $3.4 \times$ $10^{7} n^{-0.5}$ years (Spitzer 1978) so, for typical dark cloud densities of $10^{3}-10^{4} \mathrm{~cm}^{-3}$, a dynamical timescale of $3.4 \times$ $10^{5}-10^{6}$ years is inferred. $\zeta$ is usually estimated from observations of the abundances of molecular species interpreted through simple chemical models. Such methods depend on the assumption that chemical equilibrium has already been reached. It is not obvious, therefore, that estimates of the cosmic ray ionization rate in regions which may be rapidly evolving are correct.

The rates of ionization have been clearly established for diffuse clouds, largely through observations of $\mathrm{OH}$ and HD (see below). In dark clouds the situation is not so clear. The standard assumption is that the same value of $\zeta$ is applicable in dark clouds as in diffuse clouds. However, even in diffuse clouds, there is now believed to be very considerable variation in $\zeta$ from source to source. Thus, in a careful study based on $\mathrm{H}_{3}^{+}$observations in $\zeta$ Persei, McCall et al. (2003), deduce that $\zeta$ is as high as $1.2 \times 10^{-15} \mathrm{~s}^{-1}$. However, Le Petit et al. (2004) find that their model reproduces observed abundances in the same source using a value of $\zeta=2.5 \times 10^{-16}$. In addition, as dark clouds are much denser and also are permeated by ordered magnetic fields there is no a priori reason to assume that $\zeta$ is the same in diffuse and dark clouds. Moreover, as indicated above, attempts to estimate $\zeta$ in dark clouds are usually based on estimators that make an assumption of chemical equilibrium.

Most matter in a molecular cloud is essentially sterile, in that stars only form from a small fraction of the mass of a cloud (Leisawitz et al. 1999). Most of this mass exists in regions of low extinction and is effectively in a photon-dominated region (Evans 1999). Thus, most diffuse clouds are either supported against gravitational collapse, or else are transient objects that are dispersed before collapse and star-formation can occur. This implies that dark, dense, clouds form from diffuse clouds and may retain some degree of the chemical identity of their precursors.

In this paper we assess the implications of the assumption of chemical equilibrium on the inferred cosmic ray ionization rate in molecular clouds which are evolving on timescales that are short compared to the time required for chemical equilibrium to be established.

\section{Estimates of the cosmic ray ionization rate, $\zeta$}

The usual method of estimating the cosmic ray ionization rate is to employ a semi-analytical, semi-empirical technique. Thus what is, in reality, a complex multi-channel chemistry is simplified to to a small number of primary loss and formation reactions. The absolute abundances, or abundance ratios, of key tracers are then interpreted through simple analytical expressions. In practise, whilst many of the chemical abundances in these expressions are directly measureable (such as those for $\mathrm{CO}, \mathrm{HCO}^{+}$and $\mathrm{H}_{3}^{+}$), others are unobservable $\left(\right.$e.g. $\mathrm{H}_{2} \mathrm{O}$ ) or else observational data does not exist. Consequently, the values used as input to the formulae are a combination of observed quantities and predictions from (often complex) chemical models. However, both the derivation of the analytical expressions and the modelled abundances used as input almost invariably depend on the assumption of chemical equilibrium. Therefore, for the reasons stated above, it is possible that they may not yield accurate values for $\zeta$.

Lepp et al. 1987 used the relationship between the abundances of (observable) $\mathrm{OH}$ and $\mathrm{H}_{3}^{+}$and the equilibrium relationship between $\mathrm{n}\left(\mathrm{H}_{3}^{+}\right)$and $\zeta$ (see below) to deduce constraints on $\zeta$ in dense, dark interstellar clouds. This analysis was based on the assumption that the photodissociation of $\mathrm{H}_{2} \mathrm{O}$ by cosmic-ray induced ultraviolet photons is a major source of $\mathrm{OH}$ in dark clouds.

In contrast, the abundances of carbon-bearing species in diffuse clouds are not sensitive to $\zeta$, and the the best estimators of $\zeta$ come from observations and models of the $\mathrm{OH}$ and HD abundances (e.g. van Dishoeck \& Black 1986; Federman et al. 1996). The entry into the oxygen chemistry is somewhat different than in dense molecular environments. The source of $\mathrm{O}^{+}$is cosmic ray ionization of hydrogen atoms and resonant charge exchange with oxygen atoms;

$\mathrm{H}^{+}+\mathrm{O} \longrightarrow \mathrm{O}^{+}+\mathrm{H}$.

This is followed by reactions with $\mathrm{H}_{2}$ and dissociative recombination

$\mathrm{O}^{+}+\mathrm{H}_{2}, \mathrm{e}^{-} \ldots \rightarrow \mathrm{OH}, \mathrm{H}_{2} \mathrm{O}$

whilst the HD formation route is

$\mathrm{H}^{+}+\mathrm{D} \longrightarrow \mathrm{H}+\mathrm{D}^{+}$

$\mathrm{D}^{+}+\mathrm{H}_{2} \longrightarrow \mathrm{HD}+\mathrm{H}^{+}$.

Thus the abundances of both $\mathrm{OH}$ and HD are directly proportional to $\zeta$. Studies using this reaction network yield a value of $\zeta \sim 10^{-17}-10^{-16} \mathrm{~s}^{-1}$ for the rate of cosmic ray ionization of $\mathrm{H}$ atoms in diffuse clouds (Federman et al. 1996) - the spread of values being partly attributable to different assumptions being made and partly due to genuine variations, which may derive from uncertainties in the temperatures and the assumed radiation fields.

If localised heating (e.g. through the action of shocks) is present, then the reaction

$\mathrm{O}+\mathrm{H}_{2} \longrightarrow \mathrm{OH}+\mathrm{H}$

can occur, in which case these methods may overestimate the value of $\zeta$.

An alternative estimator is based on the $\mathrm{H}_{3}^{+}$abundances. In diffuse clouds, $\mathrm{H}_{3}^{+}$is formed by reactions (1) and (2) and lost by dissociative recombination:

$\mathrm{H}_{3}^{+}+\mathrm{e}^{-} \longrightarrow \mathrm{H}_{2}+\mathrm{H}$ 
although this approach can be undermined by uncertainties in the value of the dissociative recombination rate co-efficient and the electron density.

In diffuse clouds the main loss channel of simple molecular species is photodissociation and the implied timescales are of the order of 300 years, significantly less than the dynamical timescales. In addition the chemistry and rate-coefficients are well-defined. For these reasons, the inferred ionization rates are believed to be reasonably robust (Hartquist \& Williams 1995). However, McCall et al. (2003) present a direct detection of $\mathrm{H}_{3}^{+}$in diffuse clouds towards $\zeta$ Per, and infer a correspondingly high cosmic ray ionization rate, 40 times larger than the "standard" value. Le Petit et al. (2004), noting that such a high ionization rate may be inconsistent with other molecular and atomic observations (especially those of $\mathrm{OH}$ and $\mathrm{HS}$ ), explain the observed abundance of $\mathrm{H}_{3}^{+}$via a two-phase model. This example illustrates the difficulty of separating the ionization rate from effects of structure and, as we discuss below, dynamical evolution.

In dark clouds, the reactive species $\mathrm{H}_{3}^{+}$provides important entries into the ion-neutral chemistry. $\mathrm{H}_{3}^{+}$is usually more easily detected in its deuterated form, $\mathrm{H}_{2} \mathrm{D}^{+}$, to which it is linked via;

$\mathrm{H}_{3}^{+}+\mathrm{HD} \leftrightarrow \mathrm{H}_{2} \mathrm{D}^{+}+\mathrm{H}_{2}$.

This reaction also leads to the deuteration of molecular ions, such as $\mathrm{DCO}^{+}$, which can be used to constrain the fractional ionization (Caselli et al. 1998).

The oxygen chemistry follows a simple route:

$\mathrm{H}_{3}^{+}+\mathrm{O} \longrightarrow \mathrm{OH}^{+}+\mathrm{H}_{2}$

$\mathrm{OH}^{+}+\mathrm{H}_{2} \longrightarrow \mathrm{H}_{2} \mathrm{O}^{+}+\mathrm{H}$

$\mathrm{H}_{2} \mathrm{O}^{+}+\mathrm{H}_{2} \longrightarrow \mathrm{H}_{3} \mathrm{O}^{+}+\mathrm{H}$

$\mathrm{H}_{3} \mathrm{O}^{+}+\mathrm{e}^{-} \longrightarrow \mathrm{OH}+2 \mathrm{H}, \mathrm{H}_{2} \mathrm{O}+\mathrm{H}, \mathrm{OH}+\mathrm{H}_{2}$.

Other major loss routes for $\mathrm{H}_{3}^{+}$are reaction with water;

$\mathrm{H}_{3}^{+}+\mathrm{H}_{2} \mathrm{O} \longrightarrow \mathrm{H}_{3} \mathrm{O}^{+}+\mathrm{H}_{2}$

followed by dissociative recombination (6), and reaction with $\mathrm{CO}$, leading to $\mathrm{HCO}^{+}$formation;

$\mathrm{H}_{3}^{+}+\mathrm{CO} \longrightarrow \mathrm{HCO}^{+}$.

The $\mathrm{HCO}^{+}$is lost through dissociative recombination;

$\mathrm{HCO}^{+}+\mathrm{e}^{-} \longrightarrow \mathrm{CO}+\mathrm{H}$

reaction with water;

$\mathrm{HCO}^{+}+\mathrm{H}_{2} \mathrm{O} \longrightarrow \mathrm{H}_{3} \mathrm{O}^{+}+\mathrm{CO}$

and charge transfer with low ionization potential elements;

$\mathrm{HCO}^{+}+\mathrm{Na}, \mathrm{Mg} \longrightarrow \mathrm{Na}^{+}, \mathrm{Mg}^{+}+\mathrm{H}+\mathrm{CO}$.

This last reaction is particularly important in determining the ionization level of dark clouds. In metal-poor dark clouds, $\mathrm{HCO}^{+}$can be the dominant ion. However, the radiative recombination rates for elemental ions, such as $\mathrm{Na}^{+}$and $\mathrm{Mg}^{+}$, are very slow so in undepeleted regions, reaction (11) can effectively result in a higher, stable, ionization level.

Williams et al. (1998) used observations of $\mathrm{C}^{18} \mathrm{O}, \mathrm{H}^{13} \mathrm{CO}^{+}$ and $\mathrm{DCO}^{+}$towards 23 low-mass cores and a steady-state chemical model to deduce a mean value of $\zeta=5 \times 10^{-17} \mathrm{~s}^{-1}$. The $\left[\mathrm{DCO}^{+}\right] /\left[\mathrm{HCO}^{+}\right]$ratio, which is relatively insensitive to $\zeta$ was used to deduce the ionization fraction. In low mass cores, the ionization can be enhanced as a result of the presence of newly-formed low mass stars (which are X-ray sources) and the penetration of external UV, if the clouds are inhomogeneous. Bergin et al. (1999) also used obervations of $\left[\mathrm{DCO}^{+}\right] /\left[\mathrm{HCO}^{+}\right]$ to infer the fractional ionization within Orion massive dark cores, where cosmic rays are believed to be the dominant source of ionization. Both studies initially considered variations of $\zeta$ within a limited range $\left(1-15 \times 10^{-17} \mathrm{~s}^{-1}\right)$. This was constrained by the condition of thermal balance at the temperatures and densities of the sources being considered, recognising that cosmic rays are the dominant source of heating in starless cores. They subsequently adopted a value of $\zeta=5 \times 10^{-17} \mathrm{~s}^{-1}$ for all their modelled sources.

However, these techniques are complicated by the fact that the fractional ionization and $\zeta$ are only partly independent of eachother, depending also on other free parameters such as the oxygen and carbon depletion, the abundance of the low ionization potential metals and the density. Caselli et al. (1998) performed a detailed study of the ionization fraction, $\mathrm{X}\left(\mathrm{e}^{-}\right)$, and $\zeta$ in dense cores. They compared simple analytical expressions for these quantities (dependent on the two abundance ratios; $\left[\mathrm{DCO}^{+}\right] /\left[\mathrm{HCO}^{+}\right]$and $\left[\mathrm{HCO}^{+}\right] /\left[\mathrm{C}^{18} \mathrm{O}\right]$ and the free parameters listed above) with the predictions of a more elaborate chemical model, evolved to steady-state, and found reasonably good agreement, particularly for $\zeta<10^{-17} \mathrm{~s}^{-1}$. In addition, they also noted that the abundance ratios do show considerable timedependence on timescales $<10^{5}$ years. As the sources that they have modelled are all quiescent cores (from Butner et al. 1995) they conclude that they are older than $10^{5}$ years and that chemical equilibrium can be assumed. However, the chemical model that they used assumed that the physical conditions are fixed. In a dynamically evolving system, the chemical timescales may be longer and the initial conditions less well constrained.

van der Tak \& van Dishoeck (2000) also modelled the $\mathrm{HCO}^{+}$and $\mathrm{H}_{3}^{+}$abundances (in steady state) on the assumption that the $\mathrm{H}_{2} \mathrm{O}$ and $\mathrm{O}_{2}$ abundances are low (due to freeze out) unless $T>100 \mathrm{~K}$, when mantle sublimation occurs. They found that the value of $\zeta$ inferred from the $\mathrm{H}_{3}^{+}$is systematically higher than that inferred from the $\mathrm{HCO}^{+}$abundances. Noting that $\mathrm{HCO}+$ traces dense gas, they speculate that the main cause of this discrepancy is the presence of intervening clouds along the line of sight, but again we postulate that a significant cause may be the non-equilibrium character of the chemistry.

As described above, in contrast to diffuse clouds, the relevant timescale in dark clouds is approximately $10^{6}$ years, which may be significantly longer than the dynamical timescale in some scenarios. Moreover, there is increasing evidence to suggest that many of the structures in the interstellar medium may be more dynamically active than had been previously believed. Thus, for example, Elmegreen (1999) and Hartman et al. (2001) have suggested that many of the translucent clumps 
detected in $\mathrm{CO}$ are transient. This has prompted plausible models of (MHD) transient structure formation (e.g. Falle \& Hartquist 2002). These models explain the presence of small, non-gravitationally bound clumps, which are found to have lifetimes of $\sim 10^{5}$ years. This timescale is significant as it is less than the time taken for the complete conversion of $\mathrm{H}$ to $\mathrm{H}_{2}$ and for chemical equilibrium to be established. The chemical implications of an initially H-rich chemistry were investigated by Rawlings et al. (2002) who identified the presence of strong chemical signatures in such circumstances. In a cloud of density $\sim 10^{4} \mathrm{~cm}^{-3}$ the dynamical (free-fall), chemical and freezeout timescales are all comparable $\left(\sim\right.$ few $\times 10^{5}$ years $)$, so that the temporal and spatial variations of molecular abundances are expected to be sensitive to the boundary conditions (Rawlings 2003).

We suggest, therefore, that in dynamically evolving regions, the origin of the ionization can be ambigious. Specifically, a significant fraction of the ionization that is inferred for dark clouds may be a "fossilised" remnant of diffuse cloud conditions. In other words the dark cloud carries chemical signatures of its more diffuse nascent state and has not yet established dark cloud chemical equilibrium. If this is true, then the inferred ionization rates for these objects may be incorrect.

\section{Chemical indicators of $\zeta$ in dark clouds}

The chemical networks within dark molecular clouds are wellknown and, in their early stages, follow a reasonably linear chain of reactions. Without recourse to complex chemical models, it is possible to identify the key formation and destruction channels for those species which are sensitive to the the value of $\zeta$. This simplification allows simple analytical expressions which allow the determination of $\zeta$ from a knowledge of the abundances of the relevant chemical species to be defined. We investigate the validity of two well-known analytical expressions for $\zeta$

1. $\zeta_{1}$, based on the abundance of $\mathrm{H}_{3}^{+}$(see e.g. Lepp et al. 1987). $\mathrm{H}_{3}^{+}$is primarily formed by cosmic ray ionization (1) and rapid reaction with $\mathrm{H}_{2}(2)$. In dense environments it is mainly lost through reactions with $\mathrm{O}, \mathrm{H}_{2} \mathrm{O}$ and $\mathrm{CO}((3),(7)$, and (8)). Thus, in equilibrium:

$$
\zeta_{1}=\frac{n\left(\mathrm{H}_{3}^{+}\right)}{X\left(\mathrm{H}_{2}\right)}\left[k_{8} X(\mathrm{CO})+k_{3} X(\mathrm{O})+k_{7} X\left(\mathrm{H}_{2} \mathrm{O}\right)\right] .
$$

2. $\zeta_{2}$, based on the $\mathrm{HCO}^{+}$abundance (see e.g. van der Tak \& van Dishoeck 2000). $\mathrm{HCO}^{+}$is formed by reaction (8) and primarily lost through dissociative recombination (9) and reaction with water (10). Thus, after substituting for $n\left(\mathrm{H}_{3}^{+}\right)$ from Eq. (12) we obtain;

$$
\begin{aligned}
\zeta_{2}= & \frac{n\left(\mathrm{HCO}^{+}\right)\left[k_{7} X(\mathrm{CO})+k_{3} X(\mathrm{O})+k_{7} X\left(\mathrm{H}_{2} \mathrm{O}\right)\right]}{k_{8} X(\mathrm{CO}) X\left(\mathrm{H}_{2}\right)} \\
& \times\left[k_{9} X\left(\mathrm{e}^{-}\right)+k_{10} X\left(\mathrm{H}_{2} \mathrm{O}\right)\right] .
\end{aligned}
$$

In these expressions, the subscripts of the rate coefficients $\left(k_{i}\right)$ refer to the reaction numbers given in the previous section, and the fractional abundance of a species, $i$, is defined to be the ratio of its number density to the total hydrogen nucleon density;

$X_{i}=\frac{n(X)}{n(\mathrm{H})+2 n\left(\mathrm{H}_{2}\right)}$.

\section{The model}

The accuracy of both estimators for $\zeta$ was investigated using a one-point model of the time-dependent chemistry. The model treats protostellar clouds as being of uniform density and temperature, both of which change with time in three distinct phases:

1. Phase I: diffuse cloud conditions $\left(n_{0}=100 \mathrm{~cm}^{-3}, T=\right.$ $100 \mathrm{~K}, \mathrm{~A}_{v 0}=0.5$ ). The system is allowed to evolve until no abundance changes by more than $0.1 \%$ in an individual time step. At this point we deem equilibrium to have been reached.

2. Phase II: spherically symmetric, homogeneous, free-fall collapse to a pre-specified terminal density $\left(n_{\max }=\right.$ $10^{5} \mathrm{~cm}^{-3}$ ). Initially, the collapse is assumed to occur isobarically, so that the temperature is inversely proportional to the number density, subject to the constraint that it does not fall below some minimum value $\left(T_{\text {dark }}=10 \mathrm{~K}\right)$. As the collapse is assumed to be both spherically symmetric and homogeneous, the extinction $A_{\mathrm{v}}$ is given by

$$
A_{\mathrm{v}}=A_{\mathrm{v} 0}\left(\frac{n}{n_{0}}\right)^{\frac{2}{3}} \text {. }
$$

3. Phase III: relaxation to chemical equilibrium in dark cloud conditions (constant density and temperature, $T_{\text {dark }}=$ $10 \mathrm{~K})$.

The chemistry includes 87 gas-phase and 36 solid-state chemical species, composed of the elements $\mathrm{H}, \mathrm{He}, \mathrm{C}, \mathrm{N}, \mathrm{O}, \mathrm{S}$ and $\mathrm{Na}$ and linked through 1265 chemical reactions. The elemental abundances are given in Table 1. These values are broadly representative of cosmic abundances, with a depletion factor of $0.5 \times$ included for carbon. (Recent observations (e.g. Meyer et al. 1997) have suggested that nitrogen and oxygen are also depleted by $50 \%$ in diffuse clouds. However, we find that including this extra depletion results in only extremely minor changes to our results.)

We have investigated two extremes of metallicity; high (as in Table 1) and low (as in Table 1, but with the abundances of $\mathrm{S}$ and $\mathrm{Na}$ (the representative low ionization potential metal) reduced by a factor of $0.01 \times$. These values are consistent with those used by Caselli et al. (1998) and others.

The rate coefficents, $k_{i}$, for key reactions $i=2,3,7,8,9$ and 10 are taken from UMIST ratefiles (Millar et al. 1991, 1997) and are given in Table 2. It should be noted, however, that since our study is concerned with the comparison of analytical indicators with modelled abundances, both of which depend on the same rate coefficients, our results are not sensitive to the adopted values of $k_{i}$.

The passage through regions of intermediate extinction is assumed to be rapid and so no attempt has been made to model the details of the photochemistry, other than through the normal 
Table 1. Elemental abundances by number, relative to hydrogen.

\begin{tabular}{c|c}
\hline \hline Species & Abundance $/ X(\mathrm{H})$ \\
$\mathrm{He}$ & 0.1 \\
$\mathrm{C}$ & $1.87 \times 10^{-4}$ \\
$\mathrm{~N}$ & $1.15 \times 10^{-4}$ \\
$\mathrm{O}$ & $6.74 \times 10^{-4}$ \\
$\mathrm{~S}$ & $8.00 \times 10^{-6}$ \\
$\mathrm{Na}$ & $2.00 \times 10^{-6}$ \\
\hline
\end{tabular}

Table 2. Rate coefficients for key reactions $\left(\mathrm{cm}^{3} \mathrm{~s}^{-1}\right)$.

\begin{tabular}{c|c}
\hline \hline$k_{2}$ & $2.08 \times 10^{-9}$ \\
$k_{3}$ & $8.0 \times 10^{-10}$ \\
$k_{7}$ & $5.9 \times 10^{-9}$ \\
$k_{8}$ & $1.7 \times 10^{-9}$ \\
$k_{9}$ & $1.1 \times 10^{-7} \times(300 / T)$ \\
$k_{10}$ & $2.5 \times 10^{-9}$ \\
\hline
\end{tabular}

dependence on $A_{\mathrm{v}}$. We do, however, assume that $\mathrm{H}_{2}$ is selfshielding against photodissociation at all times, whilst photoionization of $\mathrm{C}$ and photodissociation of $\mathrm{CO}$ can only occur in diffuse cloud (phase I) conditions.

Other than $\mathrm{H}_{2}$ formation (for which we assume $k_{\text {dust }}=$ $1.6 \times 10^{-17} \mathrm{~cm}^{3} \mathrm{~s}^{-1}$, Rawlings et al. 1992), in the standard models we have only considered gas-phase chemistry. However, in some models we have also allowed gas-phase species to freeze out onto the surface of the grains once the extinction rises above some critical threshold value $\left(A_{v, \text { crit. }} \sim 3\right)$. As the sources are dense and cold we do no need to consider the effects of surface chemistry as the return of species contained in ice mantles to the gas-phase is unlikely to be efficient.

\section{Results}

\subsection{Evolution with time}

The evolution of selected species for a typical run is shown in Fig. 1. This figure clearly shows how the the chemistry starts from primarily atomic values appropriate for diffuse cloud conditions. As the cloud collapses and becomes denser and more opaque to photodissociating radiation, the chemistry adjusts to a more molecule-rich, lower ionization state characteristic of dark clouds.

In order to assess the validity of the analytical estimators of $\zeta$, we calculate the logarithmic ratio of the rates inferred from the two analytical expressions $\left(\zeta_{1}, \zeta_{2}\right)$ to the "true" value used in the chemical models $\left(\zeta_{\text {true }}\right)$ :

$R_{i}=\log _{10}\left(\frac{\zeta_{i}}{\zeta_{\text {true }}}\right)$

and we plot this value as a function of time. Thus, if $\zeta_{i}$ were to give a perfectly accurate value of $\zeta$ then $R_{i}$ would be zero.

Results are given in Fig. 2 for several values of $\zeta_{\text {true }}\left(10^{-18}\right.$, $10^{-17}, 10^{-16}, 10^{-15}$, and $10^{-14} \mathrm{~s}^{-1}$ ).

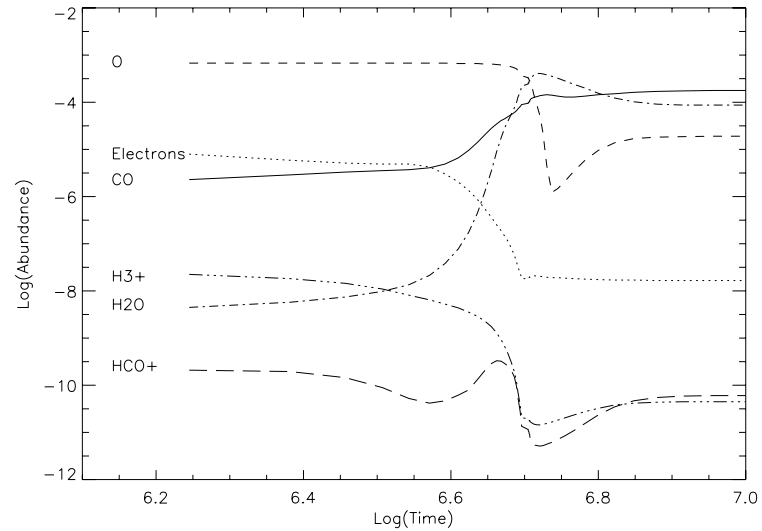

Fig. 1. The evolution of the fractional abundances, relative to hydrogen, of key species used in the determination of the cosmic ray ionization rate. In this model, the cosmic ray ionization rate is $\zeta_{\text {true }}=1 \times$ $10^{-17} \mathrm{~s}^{-1}$, and the final temperature and density are $10 \mathrm{~K}$ and $10^{5} \mathrm{~cm}^{-3}$ respectively. Collapse ends approximately at $\log ($ time $/$ years $)=6.6$.
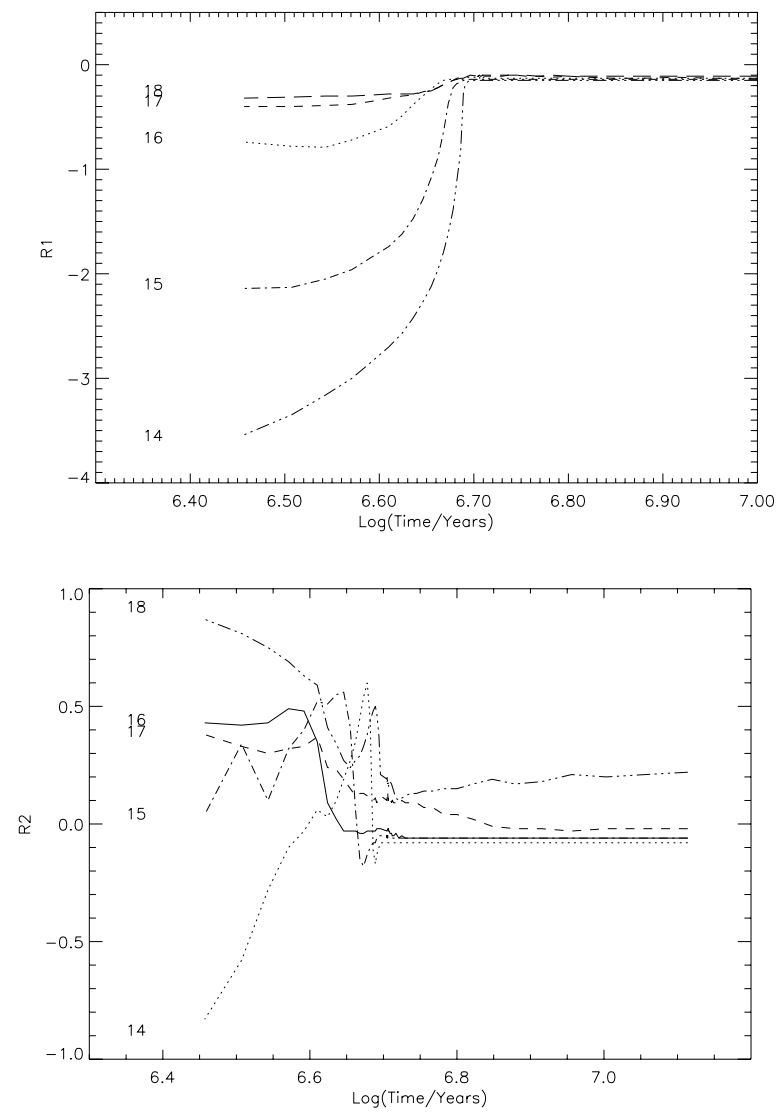

Fig. 2. The variation of a) $R_{1}$, and b) $R_{2}$ with time during phases II (collapse) \& III (post-collapse) for several values of the cosmic ray ionization rate $\left(\zeta_{\text {true }}\right)$. The labels on each curve correspond to $-\log \left(\zeta_{\text {true }}\right)$. Note that $R_{1}$ has a much larger range than $R_{2}$.

The first thing to note about these results is that, at late times (in phase III, once dynamical activity has ceased) the curves all converge to values that are very close to 0 . This is rather reassuring in the sense that (although the estimators are based on huge simplifications of the chemical networks) the expressions are remarkably accurate indicators of the cosmic ray 
ionization rate for a wide range of $\zeta$ when chemical equilibrium and dynamical steady state can be assumed.

The second point to note is that whilst there are clearly very large discrepancies during the earlier (collapse) phase, the expressions give accurate results almost as soon as the collapse phase is halted, with no notable relaxation period.

However, during the collapse (phase II) we can see that significant discrepancies are present; $\zeta_{1}$ consistently underestimates $\zeta_{\text {true }}$, but for the lower ionization rates, this is only by a factor of $\sim 2-3$. However, if $\zeta_{\text {true }}$ were as high as $10^{-16}-10^{-15} \mathrm{~s}^{-1}$, then the factor is much larger $\sim 10-10^{3} . \zeta_{2}$, which is derived incorporating additional reactions in the same network used to derive $\zeta_{1}$, is extremely sensitive to the abundances of species such as $\mathrm{H}_{2} \mathrm{O}, \mathrm{HCO}^{+}$, and especially $\mathrm{CO}$ (see Eq. (13)). These abundances change dramatically during the collapse phase. The complex structure visible in Fig. $2 b$ is partly attributable to this chemical complexity and is partly a result of inaccuracies in the numerical integration. However, as with $\zeta_{1}$, the magnitudes of the discrepancies are largest in the ealiest stages of collapse. But, in contrast to $\zeta_{1}, R_{2}$ spans a smaller range ( $\sim-1$ to 1$)$ for the values of $\zeta_{\text {true }}$ that were investigated. Also we can see that the smaller values of $\zeta_{\text {true }}$ $\left(<10^{-16} \mathrm{~s}^{-1}\right)$ are overestimated by $\zeta_{2}$, whilst the larger values of $\zeta_{\text {true }}$ are underestimated. From these results it would seem that $\zeta_{2}$ would always be $\gtrsim 10^{-17} \mathrm{~s}^{-1}$, even though $\zeta_{\text {true }}$ may be somewhat smaller.

\subsection{Sensitivity to the free parameters}

We have investigated the sensitivity of our results to the various free parameters in our model. These include the terminal density $\left(n_{\max }\right)$, the terminal temperature $\left(T_{\text {dark }}\right)$, the choice of initial conditions corresponding to diffuse cloud equilibrium or atomic abundances, whether or not freeze-out processes are included, the metallicity, and the rate of collapse in phase II.

In models where the final density $\left(n_{\max }\right)$ was varied by up to two orders of magnitude, $R_{1}$ was slightly closer to zero for the smaller values of $\zeta_{\text {true }}$, whilst $R_{2}$ was found to be quite insensitive to $n_{\max }$.

A range of post-collapse temperatures $\left(T_{\text {dark }}\right)$ between 5 and $20 \mathrm{~K}$ were also investigated for a test case with $\zeta_{\text {true }}=$ $10^{-16} \mathrm{~s}^{-1}$. The effects on $R_{1}$ were small $(\sim 20 \%)$ and are restricted to the early stages of collapse. In these early stages $R_{1}$ is larger (closer to zero) whereas the opposite effect is noted for $R_{2}$. The dependence on $T_{\text {dark }}$ is even more marginal and is also restricted to early times, but here it is the lower temperatures that result in $R_{2}$ being closer to zero.

It has been shown (Rawlings et al. 2002) that the timedependence of the chemistry may be sensitive to the assumed initial conditions - and in particular the initial $\mathrm{H}: \mathrm{H}_{2}$ ratio. In our standard model we assume that the gas is initially characterized by diffuse cloud conditions, with all hydrogen in molecular form. To investigate the possible effects of differing initial conditions on the validity of the $\zeta$ estimators we have also performed calculations where the gas is assumed to be intially atomic $\left(\mathrm{H}, \mathrm{He}, \mathrm{C}^{+}, \mathrm{N}, \mathrm{O}, \mathrm{S}^{+}\right.$and $\left.\mathrm{Na}^{+}\right)$with no $\mathrm{H}_{2}$.

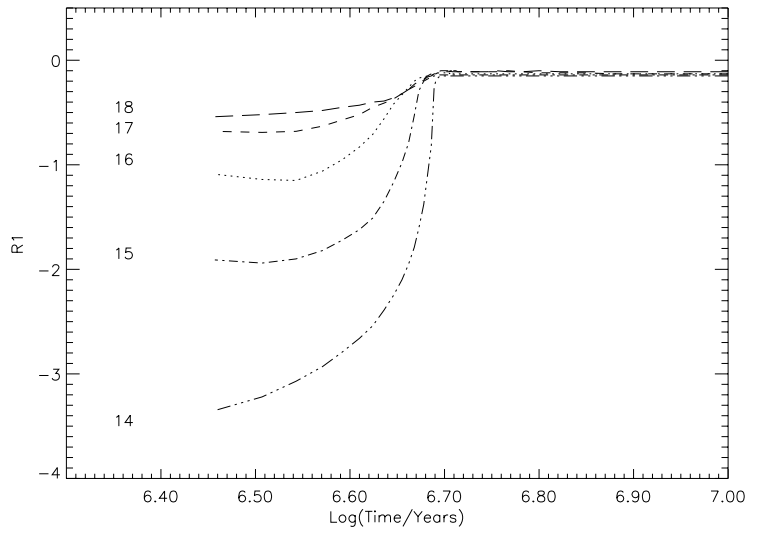

Fig. 3. Variation of $R_{1}$ with time for a model in which the chemical initial conditions (Phase I) are characterized by an atomic gas with no $\mathrm{H}_{2}$ present. As before, the labels on each curve correspond to $-\log \left(\zeta_{\text {true }}\right)$.

We find that $R_{2}$ is hardly affected by the choice of initial conditions, but some differences are noted for $R_{1}$. Results are shown in Fig. 3. For the larger values of $\zeta_{\text {true }}, \zeta_{1}$ is larger by a factor of $\sim 10 \times$ - bringing it closer to zero, in the early stages of phase II. For smaller values of $\zeta_{\text {true }}\left(<10^{-15} \mathrm{~s}^{-1}\right)$ the differences are rather marginal.

Estimates of $\zeta$ and the fractional ionization are closely linked, and the latter will be coupled to the assumption that are made concerning the metallicity of the gas. Therefore, in addition to our standard abundances we have considered a "low metallicity" model, in which the abundances of the two low ionization potential metals ( $\mathrm{S}$ and $\mathrm{Na}$ ) are reduced by a factor of 100 to $8 \times 10^{-8}$ and $2 \times 10^{-8}$ respectively.

The results show a remarkable insensitivity to the metallicity and, as noted above, only a relatively small dependence on the initial conditions. This helps confirm the hypothesis investigated by this study; that the $\zeta$ estimators used are more sensitive to the dynamical status of the observed sources than any other individual chemical or physical parameter.

To simulate accelerated collapse (Lintott et al. 2005) we artificially increased the rate of change of the density by a factor of two. This also had very minimal effects, which further supports the idea that the observed discrepancies originate from near-instantaneous dynamical effects rather than the existence of a longer-term chemical hysterisis. Other factors investigated were the extinction, and the temperature of the cloud prior to collapse. Neither had a significant effect.

The result obtained from these parameter variations is therefore that $R_{1}$ and $R_{2}$ are relatively insensitive to the various free parameters and are primarily determined by the dynamical activity within the cloud.

In our standard model we did not include any form of gasgrain interaction, but we have also investigated the effects of freeze onto the surface of the dust grains by allowing gas-phase species to freeze-out once $A_{\mathrm{v}}>3.0 \mathrm{mag}$ - similar to the critical value of the extinction required for the presence of ice water bands in Taurus (Whittet et al. 2001). This means that freezeout commences during the collapse phase (II). No desorption 

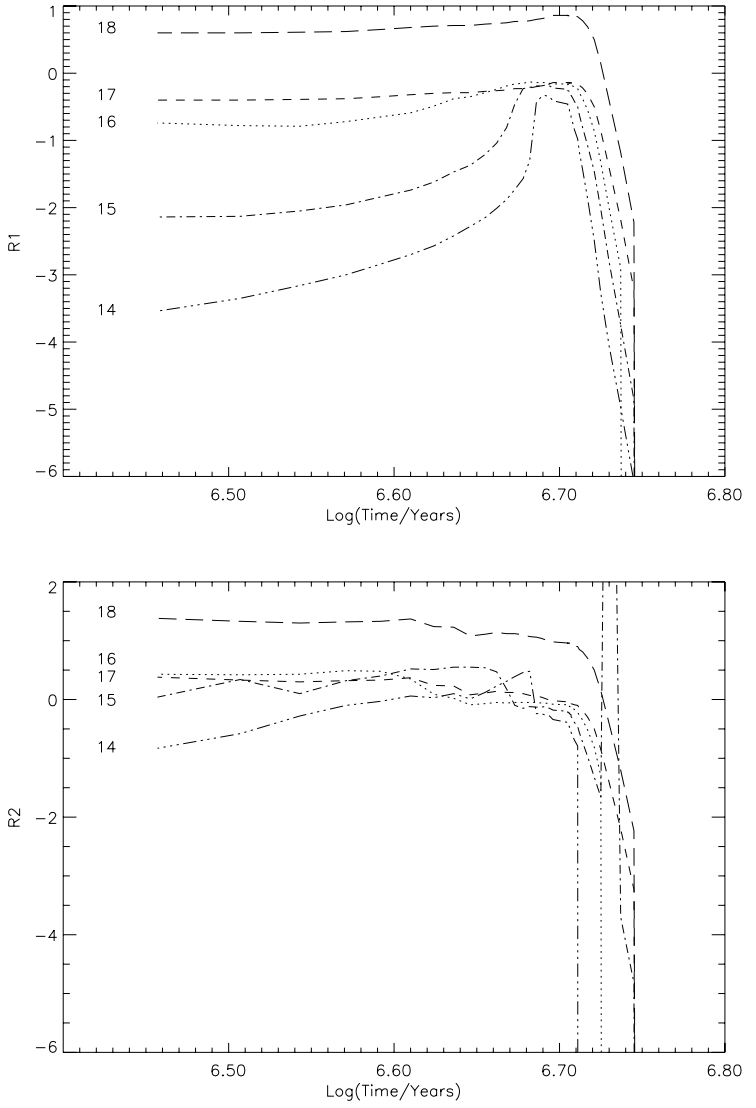

Fig. 4. Variation of a) $R_{1}$ and b) $R_{2}$ with time for a model in which freeze-out occurs once $A_{\mathrm{v}}>3.0 \mathrm{mag}$. As before, the labels on each curve correspond to $-\log \left(\zeta_{\text {true }}\right)$, and the same line style has been used for each $\zeta_{\text {true }}$ in Fig. 4b. The large jumpin $R_{2}$ at $\log ($ time $/$ years $)=6.74$ is an effect of numerical integration.

mechanisms have been included. Results are shown for both $R_{1}$ and $R_{2}$ in Fig. 4.

Two clear conclusions can be drawn from this figure: firstly, if gas-grain processes are operating - as is likely in the denser regions of the interstellar medium - then the analytical estimators always yield inaccurate values for $\zeta$, and secondly, the origins of the discrepancies (deviations of $R_{i}$ from 0 ) are separable. In the collapse phase the differences have a dynamical origin, as in the gas-phase models, whilst in the static, postcollapse phase the differences are entirely a consequence of the freezing out of molecules onto dust grain surfaces.

\section{Discussion and conclusions}

Two related methods of estimating the true ionization rate, $\zeta_{\text {true }}$, have been tested for a wide range of parameters. These were based on chemical networks centered on reactions of $\mathrm{H}_{3}^{+}\left(\zeta_{2}\right.$ using an extended version of the network used by $\zeta_{1}$ ). In those cases where gas-grain interactions were ignored, we found that chemical equilibrium is rapidly re-established after the end of collapse, and both analytical expressions give accurate measures of the true cosmic ray ionization rate in the absence of dynamical activity. If freeze-out processes are important, then the analytical expressions are inaccurate at all times.
There are two components to the modelled discrepancy between the analytical expressions for $\zeta_{1}, \zeta_{2}$ and the true cosmic ray ionization rate $\zeta_{\text {true }}$. Firstly, the fact that the analytical expressions $\left(\zeta_{1}\right.$ and $\left.\zeta_{2}\right)$ are simplifications of a rather complex chemistry and only make allowance for the dominant chemical pathways, and secondly deviations from the assumption of equilibrium. Of course it is the latter that is the subject of this paper but, as noted in the previous section, it is reassuring to see that - at late times - the simple analytical expressions are reasonably accurate and the contribution from the former is relatively small. However, this is not always the case and has been investigated in some detail by Caselli et al. (1998) (see their Fig. 9) who concluded that, even in an equilibrium approximation, the analytical expressions may overestimate $\zeta$ by a factor of $\sim 2-3$ for the cases where $\zeta>10^{-17} \mathrm{~s}^{-1}$.

In our models we (obviously) use the same set of rate co-efficients in the analytical expressions and the full timedependent models. Therefore, as the results are presented as the ratios of these quantities, they are reasonably robust and insensitive to the values of the adopted rate co-efficients.

In all of the cases that we investigated, however, we found that the analytical expressions are very inaccurate in rapidly evolving, dynamically active regions. Discrepancies of several orders of magnitude are present, especially for the largest values of $\zeta_{\text {true }}$. Such conditions may, indeed, be appropriate in many dark clouds that have previously been thought of as quiescent entities. In support of this hypothesis, it should be noted that there is an increasing body of evidence that suggets that dark molecular clouds are not monolithic entities, but consist of ensembles of clumps and sub-structure that are not resolved with single-dish molecular line observations. For example, populations of distinct sub-cores have been seen in TMC-1 Core D (Peng et al. 1998) and L673 (Morata et al. 2003). Moreover, most of these cores are not self-gravitating, but are probably transient entities or varying size/mass and dynamical status. This picture has been supported by recent chemical models (Garrod et al. 2005) which identify the sub-cores as objects that grow and decay on timescales of $\sim 10^{6}$ years.

Some sensitivity to physical parameters was found, in particular to the density reached after collapse. However, these effects were much smaller than the differences observed in the same core at different stages of collapse. It can therefore be seen that these methods cannot accurately determine the cosmic ray ionization rate unless collapse can be definitely shown to have been completed. This forms a challenging observational requirement.

Geballe et al. (2003) use the observed $\mathrm{H}_{3}^{+}$abundance in several dark clouds to constrain the cosmic ray ionization rate. Oka et al. (2005) compare these measurements of cosmic ray ionization to their measurements of $\zeta$ in diffuse clouds. However, the results presented in this paper suggest that such a comparison is invalid unless the dark clouds being studied were sufficiently old for the dynamical effects revealed by our models to be unimportant.

In their study of massive star-forming regions, van der Tak $\&$ van Dishoeck (2000) found that the cosmic ray ionization rate as deduced from $\mathrm{H}_{3}^{+}$observations was greater than that as deduced from $\mathrm{HCO}^{+}$observations. That is to say, models 
using $\zeta$ derived from $\mathrm{H}_{3}^{+}$observations resulted in a significant over-production of $\mathrm{HCO}^{+}$. They speculated that, whilst the $\mathrm{HCO}^{+}$measure probes only the dense molecular gas, contributions from foreground layers (e.g. in photon dominated regions or translucent clouds) may be responsible for the discrepancies. This is a valid explanation, but we note that dynamical activity may help explain the discrepancies; the differences between the values of $\zeta$ inferred from the $\mathrm{H}_{3}^{+}$and the $\mathrm{HCO}^{+}$observations (effectively quantified by $R_{1}-R_{2}$ ) depends on the "true" value of $\zeta$ and the dynamical status of the observed source. The more dynamically active the source, the greater the discrepancy.

In conclusion, it is possible that, if a reasonably full dataset of observations of key molecular tracers is available for any given source, the absolute values of $\zeta_{1}$ and $\zeta_{2}$ and the difference between $R_{1}$ and $R_{2}$ (and perhaps other analytical expressions for $\zeta$ ) could be used to simultaneously constrain the true ionization rate and the dynamical status of the source. If it turns out that very similar values are obtained for $\zeta_{1}$ and $\zeta_{2}$ then that is an indication that the observed cores are chemically "old" and dynamically quiescent.

Acknowledgements. C.J.L. is supported by a PPARC studentship.

\section{References}

Bergin, E. A., \& Langer, W. D. 1997, ApJ, 486, 316

Bergin, E. A., Plume, R., Williams, J. P., \& Myers, P. C. 1999, ApJ, 512,724

Butner, H. M., Lada, E. A., \& Loren, R. B. 1995, ApJ, 448, 207

Caselli, P., Walmsley, C. M., Terzieva, R., \& Herbst, E. 1998, ApJ, 499, 234

Elmegreen, B. G. 1999, ApJ, 527, 266

Evans, N. J. 1999, ARA\&A, 37, 311

Falle, S. A. E. G., \& Hartquist, T. W. 2002, MNRAS, 329, 195

Federman, S. R., Weber, J., \& Lambert, D. L. 1996, ApJ, 463, 181

Garrod, R. T., Williams, D. A., \& Rawlings, J. M. C. 2005, ApJ, submitted

Geballe, T., McCall, B. J., \& Oka, T, 2003, Chemistry as a Diagnostic of Star Formation, ed. C. Curry, \& M. Fich (Ottawa: MRC Press)

Goldsmith, P. F., \& Langer, W. D. 1978, ApJ, 222, 881
Hartquist, T. W., \& Williams, D. A. 1995, The Chemically Controlled Cosmos, CUP, Cambridge

Hartquist, T. W., Rawlings, J. M. C., Williams, D. A., \& Dalgarno, A. 1993, QJRAS, 34, 213

Hartmann, L., Ballesteros-Paredes, J., \& Bergin, E. A. 2001, ApJ, 562, 852

Leisawitz, D., Bash, F. N., \& Thaddeus, P. 1999, ApJS, 70, 737

Le Petit, F., Roueff, E., \& Herbst, E. 2004, A\&A, 417, 993

Lepp, S., Dalgarno, A., \& Sternberg, A. 1987, ApJ, 321, 383

Li, Z.-Y., Shematovich, V. I., Wiebe, D. S., \& Shustov, B. M. 2002, ApJ, 569, 792

Lintott, C. J., Viti, S., Rawlings, J. M. C., et al. 2005, ApJ, 620, 795

McCall, B. J., Geballe, T. R., Hinkle, K. H., \& Oka, T. 1999, ApJ, 522, 338

McCall, B. J., Huneycutt, A. J., Saykally, R. J., et al. 2003, Nature, 422, 500

Meyer, D. M., Cardelli, J. A., \& Sofia, U. J. 1997, ApJ, 490, 103

Millar, T. J., Rawlings, J. M. C., Bennett, A., Brown, P. D., \& Charnley, S. B. 1991, A\&AS, 87, 585

Millar, T. J., Farquhar, P. R. A., \& Willacy, K. 1997, A\&AS, 121, 139

Morata, O., Girart, J. M., \& Estalella, R. 2003, A\&A, 397, 181

Oka, T., Geballe, T. R., Goto, M., Usuda, T., \& McCall, B. T. 2005 , AJ, 632, 882

Peng, R., Langer, W. D., Velusamy, T., Kuiper, T. B. H., \& Levin, S. 1998, ApJ, 497, 842

Rawlings, J. M. C., Hartquist, T. W., Menten, K. M., \& Williams, D. A. 1992, MNRAS, 255, 471

Rawlings, J. M. C., Hartquist, T. W., Williams, D. A., \& Falle, S. A. E. G. 2002, A\&A, 391, 681

Rawlings, J. M. C. 2003, Ap\&SS, 285, 777

Ruffle, D. P., Hartquist, T. W., Rawlings, J. M. C., \& Williams, D. A. 1998, A\&A, 334, 678

Spitzer, L., Jr. 1978, Physical Processes in the Interstellar Medium (New York: Wiley), 282

Meyer, D. M., Cardelli, J. A., \& Sofia, U. J. 1997, ApJ, 409, 103

van der Tak, F., \& van Dishoeck, E. F. 2000, A\&A, 358, L79

van Dishoeck, E. F., \& Black, J. H. 1986, ApJS, 62, 109

Ward-Thompson, D., Nutter, D., Kirk, J., \& Andre, P. 2004, ApJ, 600, 279

Whittet, D. C. B., Gerakines, P. A., Hough, J. H., \& Shenoy, S. S. 2001, ApJ, 547, 872

Williams, J. P., Bergin, E. A., Caselli, P., Myers, P. C., \& Plume, R. 1998, ApJ, 503, 689 